\title{
Spatial analysis of the frequency-magnitude distribution and decay rate of aftershock activity of the 2000 Western Tottori earthquake
}

\author{
Bogdan Enescu ${ }^{1,2}$ and Kiyoshi Ito ${ }^{1}$ \\ ${ }^{1}$ Research Center for Earthquake Prediction, Disaster Prevention Research Institute, Kyoto University, Gokanosho, Uji, Kyoto 611-0011, Japan \\ ${ }^{2}$ National Institute for Earth Physics, P.O. Box MG-2, 76900, Bucharest-Magurele, Romania
}

(Received September 12, 2001; Revised February 25, 2002; Accepted February 25, 2002)

\begin{abstract}
The $b$-value of the frequency-magnitude distribution and the parameters in the modified Omori law, describing the decay rate of aftershock activity, are investigated for more than 4000 aftershocks identified in the first four months after the Western Tottori earthquake (October 6, 2000). We used the JMA data catalog, containing aftershocks with magnitude larger than or equal to 2.0. The studied area is first divided into three areas: one region (A) corresponding to the main aftershock area and other two (B and C) corresponding to seismic activity probably triggered by the stress change caused by the main shock. For region A, the magnitude of completeness (Mc) decreases with time, from the largest value of 3.2 in the first two hours of the sequence, to 2.0, about four days after the main shock. Taking the threshold magnitude as 3.2, we estimated the $b$-value for the whole region A to be about 1.3 and $p$-value around 1 . However, highly significant variations in both $b$ and $p$ values are found when analyzing their spatial distribution in region $\mathrm{A}$. The seismic activity in the regions $\mathrm{B}$ and $\mathrm{C}$ started about 2.5 days after the main shock. The $b$-value for region $\mathrm{B}(\mathrm{Mc}=2)$ is 1.05 . The decay rate of earthquake activity in Region B is well modeled by the modified Omori law and the $p$-value is found to be relatively low (0.83). The number of events in region $\mathrm{C}$ is too small for a meaningful study. The physical interpretation of the spatial variation of the parameters is not straightforward. However, the variation of $b$-value can be related to the stress distribution after the main shock, as well as the history of previous ruptures. Thus, the relatively low stress in the regions that have already experienced rupture is probably responsible for the larger value of $b$ found in these areas. Regions with relatively low $b$-value, on the other hand, are probably regions under higher applied shear stress after the main shock. Alternatively, one can hypothesize that the areas that experienced slip are more fractured, favoring higher $b$-values. The larger $p$-values correlate well with the regions that experienced larger slip during the main shock, while small $p$-values are found generally in regions that have not ruptured recently. The variation of $p$-value can be related with the frictional heating produced during rupture. The crustal structure may explain some local features of $b$ and $p$ value spatial distribution. In order to verify our hypothesis we also analyzed the seismic activity that occurred before the Tottori earthquake, starting in 1978, using the data of DPRI, Kyoto University. It seems that the previous seismic activity associated with some moderate events in 1989, 1990 and 1997 had an influence on the following seismicity in the area-in particular on the spatial distribution of $b$ and $p$ values observed for the aftershocks of the Tottori earthquake. The aftershocks of the 1997 M5.5 earthquake have a larger $p$-value than previous aftershock sequences, while the $b$-value has a clear increase following the M5.5 event.
\end{abstract}

\section{Introduction}

Aftershock sequences offer a rich source of information on the Earth's crust and source properties of large earthquakes, because a very large number of events occur over a short period of time in a small area. Aftershocks also pose a significant seismic hazard, which under certain circumstances can even exceed the main shock hazard. Two basic relations describe the aftershock activity, the GutenbergRichter law, which describes the power-law size distribution of earthquakes, and the modified Omori law, which describes the decay of the aftershock activity. The frequencymagnitude distribution (Gutenberg and Richter, 1944) describes the relationship between the frequency of occurrence

Copy right (C) The Society of Geomagnetism and Earth, Planetary and Space Sciences (SGEPSS); The Seismological Society of Japan; The Volcanological Society of Japan; The Geodetic Society of Japan; The Japanese Society for Planetary Sciences. and magnitude of earthquakes:

$$
\log _{10} N=a-b M
$$

where $N$ is the cumulative number of events having magnitude larger than and equal to $M$, and $a$ and $b$ are constants. The $b$-value has been observed to vary spatially as well as temporally. Recently, several studies revealed spatial variations in the frequency-magnitude distribution in various tectonic regimes (Wiemer and Benoit, 1996; Wyss et al., 1997; Wiemer and Wyss, 1997), by using high-quality earthquake catalogs and a technique of high-resolution spatial mapping of the frequency-magnitude distribution.

Many factors can cause perturbations in $b$ from its standard value of about one. Increased material heterogeneity results in high $b$-values (Mogi, 1962a), while an increase in the shear stress or decrease in the confining pressure (Scholz, 1968, Wyss, 1973) decrease the $b$-value. In ad- 


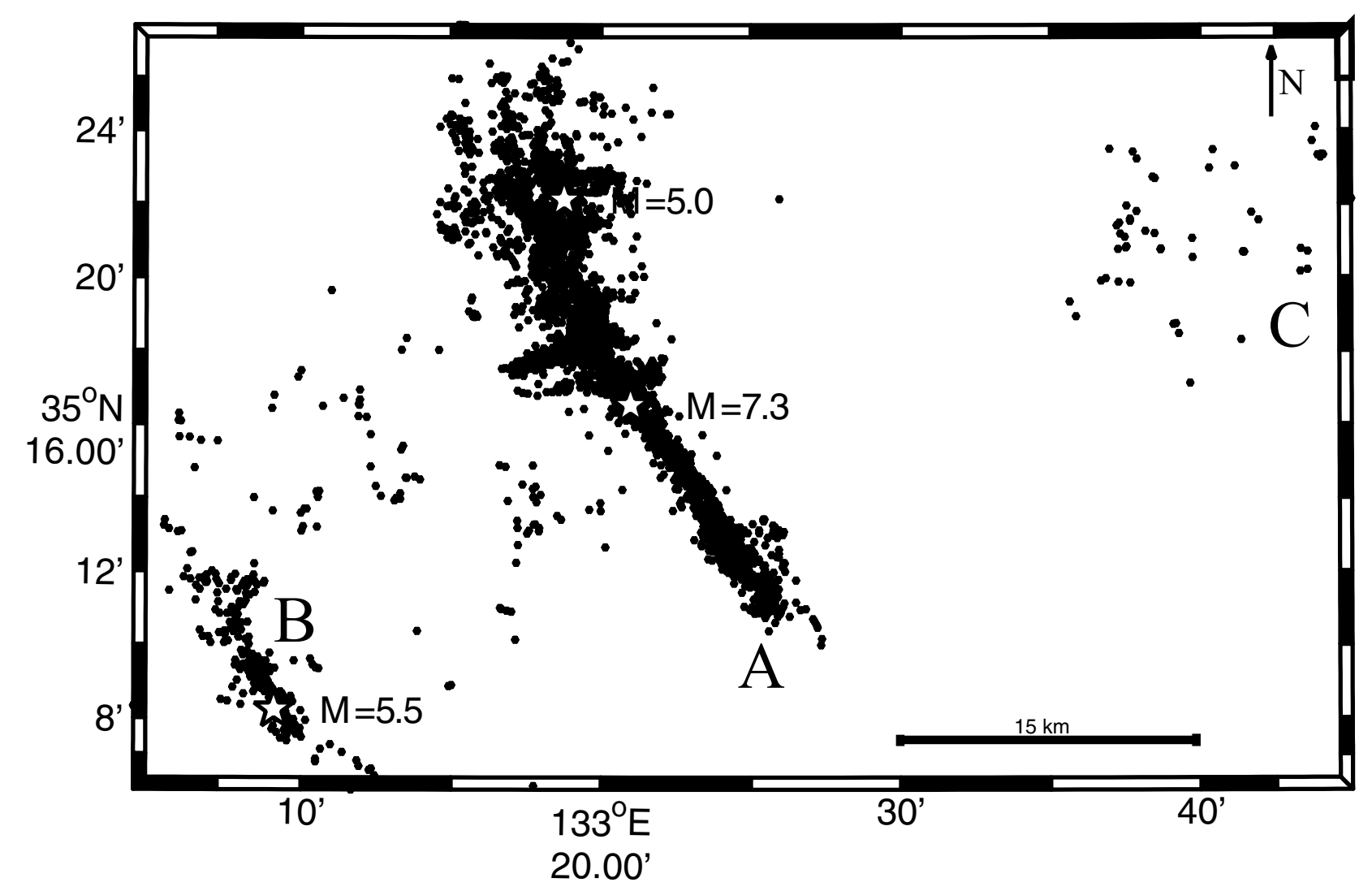

Fig. 1(a). Epicenters of the aftershocks of the Western Tottori earthquake. A, B and C regions are referred in the text. The epicenters of the main shock and of the largest aftershocks are marked by stars.

dition, an increase in the thermal gradient may cause an increase in $b$-value (Warren and Latham, 1970).

The occurrence rate of aftershock sequences in time is empirically well described by the modified Omori formula (Utsu, 1957, 1961):

$$
n(t)=\frac{K}{(t+c)^{p}}
$$

where $n(t)$ is the frequency of aftershocks per unit time, at time $t$ after the main shock. $K, c$ and $p$ are constants. The characteristic parameter $p$ usually changes in value from 0.9 to 1.5 , regardless of the cutoff magnitude. The variability in $p$-value may be related to the structural heterogeneity, stress and temperature in the crust (Mogi, 1962b; Kisslinger and Jones; 1991, Utsu et al., 1995). However, it is not yet clear which of these factors is most significant in controlling the $p$-value. The $K$-value is dependent on the total number of events in the sequence and $c$ on the rate of activity in the earliest part of the sequence. The constant $c$ is a controversial quantity (Utsu et al., 1995). It is strongly influenced by the incompleteness of the catalog in the early part of a sequence. Careful analyses showed that the $c$-value is non-zero at least for some aftershock sequences. The parameters in the modified Omori formula can be estimated accurately by the maximum likelihood method, assuming that the seismicity follows a non-stationary Poisson process (Ogata, 1983).

Here, we present a detailed analysis of the $b$-value of the frequency-magnitude distribution and the $p$-value of the modified Omori law, for more than 4000 earthquakes, iden- tified in the first four months after the M7.3 (on JMA scale) Western Tottori earthquake that occurred on October 6, 2000 in southwest Japan. In the first part of our study, we analyze the variation of parameters in broad areas, while in the second part a detailed spatial analysis is performed. The third part of the study analyzes the seismic activity before the Tottori earthquake, in order to understand better the physical mechanisms of the variations of $b$ and $p$ values. The results we present are important not only for the source process of the Western Tottori aftershock sequence itself, but also for the basic understanding of the aftershock phenomenon. Previous studies found considerable spatial variability of $b$ and $p$ for different aftershock sequences (Kisslinger and Jones, 1991; Guo and Ogata, 1997). Wiemer and Katsumata (1999) found significant spatial variation within one aftershock sequence for Landers, Northridge, Morgan Hill and Kobe aftershocks. More work has to be done, however, in order to understand the variation patterns and their underlying physical mechanism.

\section{Data Used and Method of Analysis}

We used the JMA (Japanese Meteorological Agency) catalog for the study of the aftershocks of the Tottori earthquake. We selected all events with $M \geq 2$ that occurred from the time of the main shock (October 6, 2000) until January 24,2001 . The epicenters are included in a rectangle defined by $133^{\circ} 05^{\prime} \mathrm{E}$ to $133^{\circ} 45^{\prime} \mathrm{E}$ and $35^{\circ} 06^{\prime} \mathrm{N}$ to $35^{\circ} 27^{\prime} \mathrm{N}$ (Fig. 1(a)). The symbol A in Fig. 1(a) indicates the main aftershock area. The main aftershock area is presented alone, 


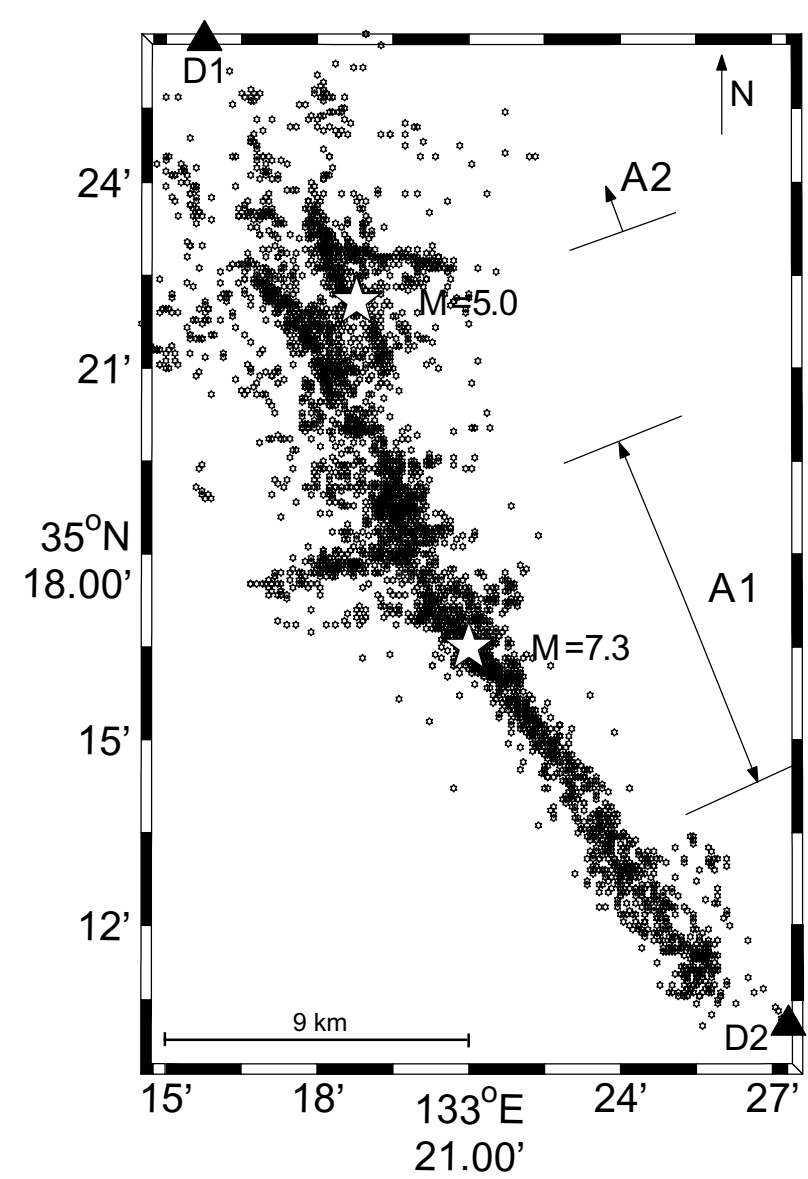

Fig. 1(b). An enlarged region A (Fig. 1(a)). A1 and A2 indicate two sub-regions for which the aftershock activity is compared (see text). D1 and D2 indicate the profile used for projection in Fig. 4. Stars mark the epicenters of the main shock and of the largest aftershock in the region.

in Fig. 1(b). B and C in Fig. 1(a) correspond to areas where the earthquake activity started about 2.5 days after the main shock. The earthquakes in these regions are not aftershocks in a strict sense; however, we considered it appropriate to include them in this analysis, since they are probably triggered by the main shock. It has been shown, by using the Coulomb failure criterion (Earthquake Prediction Information Division, JMA, 2001), that these events occurred in regions where the static stress (stress associated with co-seismic fault displacements) increased due to the main shock. As we will see in the next section, the seismic activity in region $B$ is well described by the modified Omori law. For these reasons, we will refer to the seismic activity in these areas as aftershocks. The examination of travel time data for earthquakes used in this study gave us confidence in the earthquake epicenters, since the events of magnitude greater than 2 have a sufficient number of $P$ and $\mathrm{S}$ wave arrival times. We used the DPRI, Kyoto University, catalog extensively in order to test whether the results are similar with those obtained from the JMA catalog. We find similar results using the two catalogs. However, we use the results from the JMA catalog, since its magnitude of completeness is smaller for the earliest part of the aftershock sequence.
For the estimate of both the $b$ and $p$ values, it is important to use a data set that is complete for all magnitude bands included in the analysis. The estimate of the magnitude of completeness (Mc) is based on the assumption of a powerlaw Gutenberg-Richter relationship and taken as the magnitude where the first derivative of the frequency-magnitude curve has its maximum. For region A, we used an overlapping moving window technique to compute $\mathrm{Mc}$ as a function of time. We choose 150 events/window, the window being moved by 15 events. Figure 2 shows the variation of Mc with time. Mc is highest at the very beginning of the sequence (around two hours), and then decreases very quickly to about 2.3. Four days after the main shock Mc reaches a stable value around 2 . This is because just after the main shock, when many aftershocks occurred, the data processing was restricted to larger events. Region B has only around 300 events; therefore the completeness magnitude is determined for all the shocks and not as a function of time. In region $\mathrm{C}$ the number of events with $\mathrm{M} \geq 2.0$ is very small (less than 50) and consequently we exclude these shocks from further analysis.

In Subsection 3.1 we present the results obtained by analyzing regions A and B (Fig. 1(a)) separately. Also, based on the difference in the characteristics of the earthquake sequences, we select two sub-regions, A1 and A2 (Fig. 1(b)), inside the main aftershock area (A) to study the individual $b$ and $p$ values in relation with the rupture process and the structure of the crust.

In order to ensure completeness in our analysis, two parameters need to be adjusted: (a) a minimum magnitude threshold $\mathrm{M}_{\min }$ and (b) a minimum time threshold $\mathrm{T}_{\text {start }}$, i.e. excluding the first several hours to days from the analysis. We checked some values and chose $\mathrm{M}_{\min }=3.2$ and $\mathrm{T}_{\text {start }}=0$ to determine the frequency-magnitude distribution and the parameters of the Omori law for regions A, A1 and A2. In this way, even though the number of earthquakes is drastically reduced, we can include in the analysis the earliest part of the sequence and assure completeness. For region $\mathrm{B}, \mathrm{M}_{\min }=\mathrm{Mc}=2.0$ and $\mathrm{T}_{\text {start }}=0$ were chosen.

Due to the large number of events in region $\mathrm{A}$, a more detailed spatial analysis is possible. The method employed is based on a gridding technique, which is described in detail at the end of this section. The obtained results are presented and discussed in Subsection 3.2. All the shocks with $M \geq 2.0$ are included except for the events in the first 17 hours (0.72 days) after the main shock. The overall magnitude of completeness has a value around 2.2. By doing this, we do not include data with severe incompleteness, but have enough remaining events for a meaningful spatial analysis. In addition, the completeness magnitude is checked spatially, as outlined below.

The $b$-value in the frequency-magnitude relation (Eq. (1)) has been determined using the maximum likelihood method (Aki, 1965; Utsu, 1965), because it yields a more robust estimate than the least-square regression method.

With large samples and slow temporal changes in $b$, the standard error of $b$ (Shi and Bolt, 1982) is,

$$
\sigma(b)=2.30 b^{2} \sigma(\bar{M})
$$




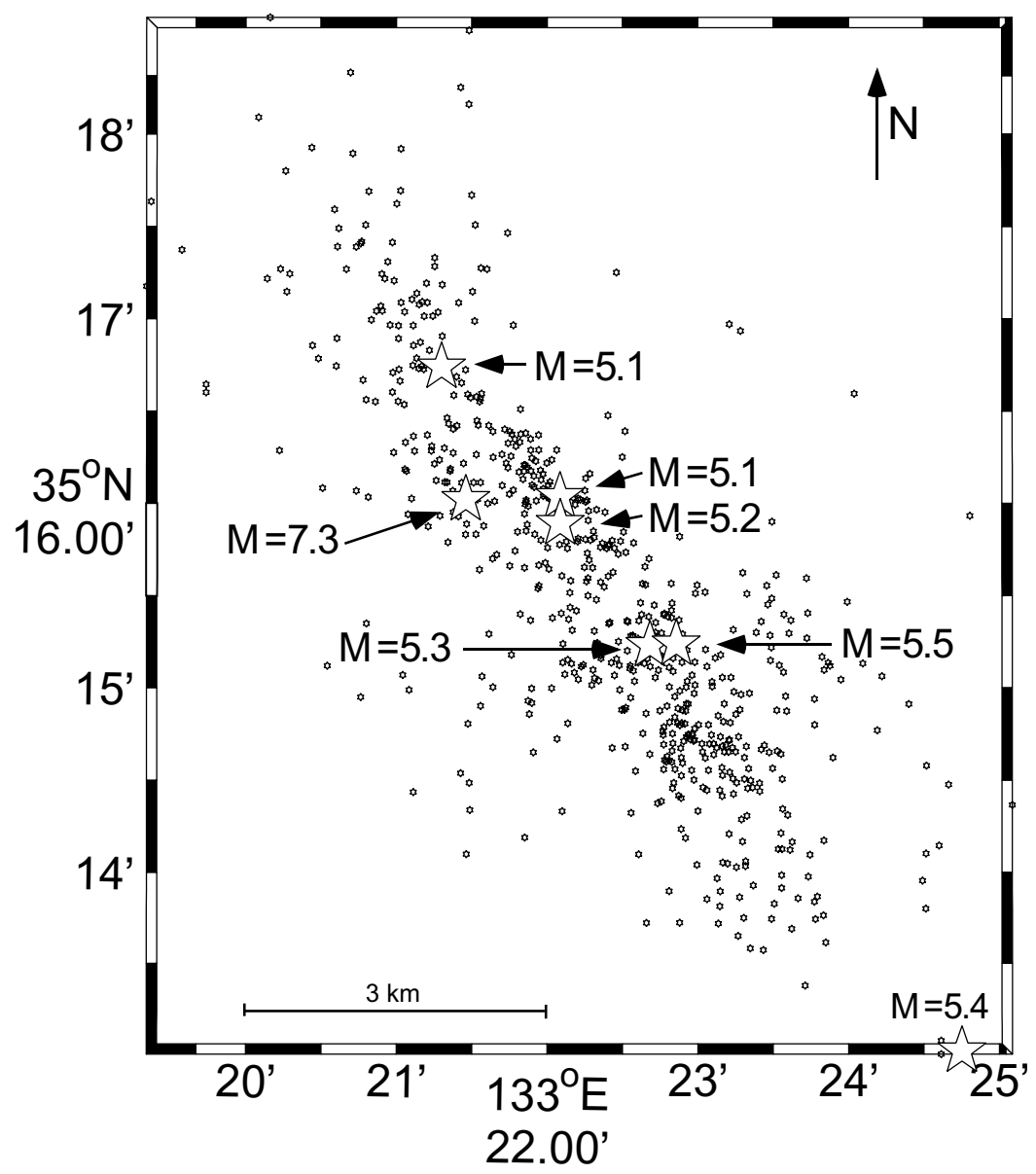

Fig. 1(c). The seismic activity $(M \geq 1.5)$ before M7.3 Tottori earthquake, from 1978 to the main shock. The epicentral area corresponds to the occurrence of several seismic swarms in 1989, 1990 and 1997. The epicenters of the main shock and of the largest aftershocks are marked by stars. Stars mark the earthquakes with $\mathrm{M} \geq 5.0$.

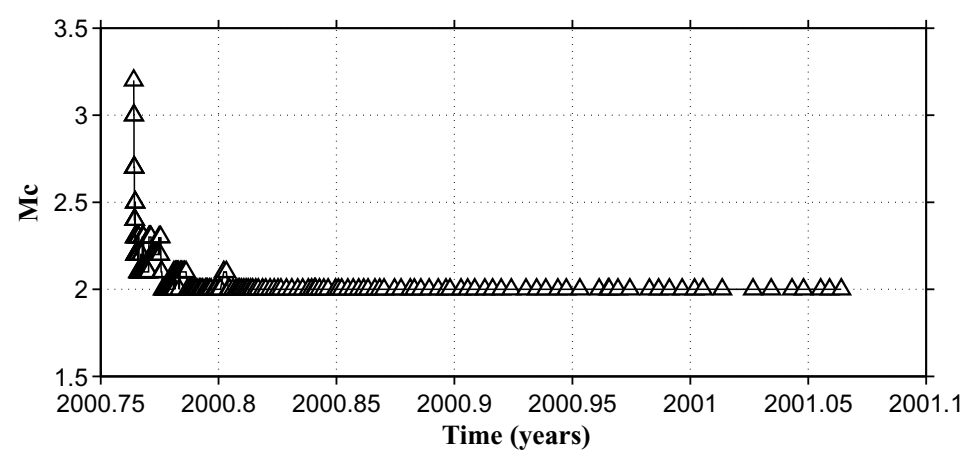

Fig. 2. Mc (magnitude of completeness) as a function of time for region A. There are 150 earthquakes in a moving-window, the window being moved by 15 events. The values of Mc are assigned at the end of the moving-window.

where

$$
\sigma^{2}(\bar{M})=\frac{\sum_{i=1}^{n}\left(M_{i}-\bar{M}\right)^{2}}{n(n-1)}
$$

$\bar{M}$ is the mean magnitude and $n$ is the sample size. The $b$-value and its standard error are computed also using a least-square method. The results obtained using the two approaches (maximum likelihood and least-square) are similar in general, indicating that the determined $b$-values do not depend on the method used.

We estimate the probability that two samples may come from the same population by Utsu's (1992) test.

$$
\operatorname{Prob} \approx \exp (-d A / 2-2),
$$

where

$$
\begin{aligned}
d A= & -2 N \ln (N)+2 N_{1} \ln \left(N_{1}+N_{2} b_{1} / b_{2}\right) \\
& +2 N_{2} \ln \left(N_{1} b_{2} / b_{1}+N_{2}\right)-2,
\end{aligned}
$$



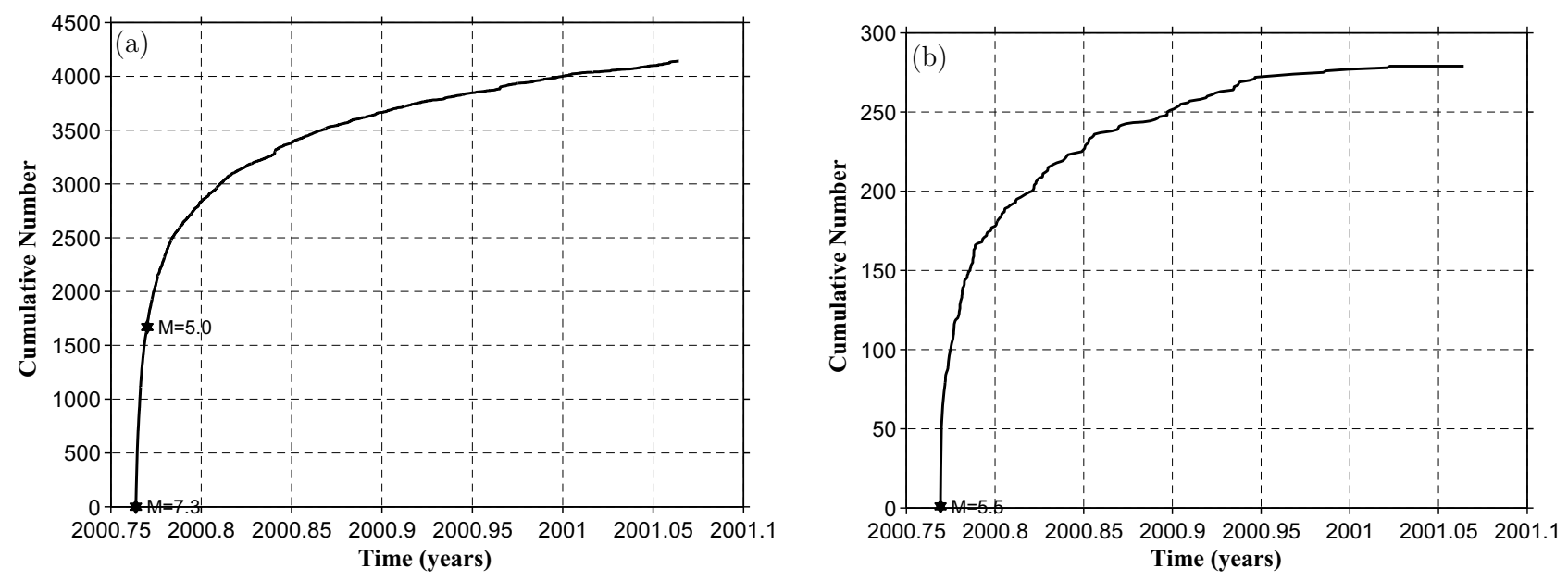

Fig. 3. Cumulative number of events versus time for all earthquakes $(M \geq 2)$ in region $A(a)$ and $B$ (b). The main shock and the largest aftershocks are marked by stars.

and

$$
N=N_{1}+N_{2}
$$

where, $N_{1}$ and $b_{1}$ are the number of earthquakes and the $b$-value for the first sample, respectively; $N_{2}$ and $b_{2}$ are the number of earthquakes and the $b$-value for the second sample, respectively.

The parameters in the modified Omori formula can be estimated accurately by the maximum likelihood method, assuming that seismicity follows a non-stationary Poisson process (Ogata, 1983). The method allows one to obtain the asymptotic standard error of the maximum likelihood estimates, based on the variance-covariance matrix of the model. To establish the significance of differences between $p$-values, we use the $95 \%$ confidence limit (1.96 standard deviations) of the difference in the standard deviations of the two $p$-values.

For all our computations, we used the computer programs ASPAR (Reasenberg, 1994) and ZMAP (Wiemer and Zuniga, 1994; Wiemer, 2001). The new release of ZMAP (version 6-freely available on Stefan Wiemer's home page, http://seismo.ethz.ch/staff/ stefan/) includes most of the routines we wrote or adapted for Matlab and used for aftershock analyses. The routines are provided with instructions and explanations on their use.

In order to visualize the frequency-magnitude distribution and the decay rate of aftershocks in region $\mathrm{A}$, we used a dense spatial grid of points and consider the nearest epicenters $(\mathrm{Ne})$ for each node of the grid. For each node we estimate the goodness of fit for the observed frequencymagnitude distribution. The algorithm (Wiemer and Wyss, 2000) then determines the minimum threshold magnitude for which the goodness of fit is greater than or equal to $95 \%$. If there is no such magnitude for the given confidence level, a $90 \%$ goodness of fit is assigned instead. If, however, the goodness of fit is less than $90 \%$ for any threshold magnitude, the magnitude where the frequency-magnitude distribution has its maximum curvature is determined. One of these magnitudes becomes the Mc for that grid point. If the number of earthquakes with $\mathrm{M} \geq \mathrm{Mc}$ is larger than or equal to $\mathrm{Ne}_{\min }, b$ and $p$ values are computed for that node by using only the earthquakes with $\mathrm{M} \geq \mathrm{Mc}$. Otherwise, the $b$ and $p$ values are not computed. Various values for the spacing of the grid and for $\mathrm{Ne}$ and $\mathrm{Ne}_{\text {min }}$ were chosen, in order to check the consistency of the results. The $b$ and $p$ values estimated in each node are translated into a color code. For a more detailed discussion of the spatial mapping of the $b$-value, refer to Wyss et al. (1997). The spatial mapping of the parameters was done by using the software ZMAP.

In the last part of our study (Subsection 3.3) we analyze the seismic activity that occurred before the Tottori earthquake, in order to get a better understanding of the variations of the pattern of $b$ and $p$ values. We have used the catalog of DPRI, Kyoto University, and selected all the events with magnitudes larger than or equal to 1.5 that occurred between 1978 and the main shock, in an area corresponding roughly with the region A1 in Fig. 1(b). The overall magnitude of completeness is 1.5 . The DPRI catalog was chosen for its better quality compared with the JMA catalog in the same period of time, but both catalogs were used for checking the compatibility of the results. Since the seismic activity before the Tottori earthquake is analyzed mainly as a function of time, the location of events is not an important issue in this case. Therefore, by choosing a lower threshold magnitude (1.5), the accuracy of the results is not affected by possible location errors.

\section{Results and Discussion}

\subsection{Characterization of the aftershock activity in broad} areas

Figures 3(a) and (b) present the cumulative number of earthquakes versus time for regions $\mathrm{A}$ and $\mathrm{B}$, respectively. One can easily see the decreasing characteristics of the aftershock sequence. The largest aftershocks in both regions are indicated by stars in Fig. 3.

Figure 4 represents the space-time distribution of the earthquakes in Region A. The projection is taken along a profile D1D2, indicated in Fig. 1(b). There are three M4.5 aftershocks very close temporally and spatially to the main shock. The other two M4.5 shocks and the largest aftershock 


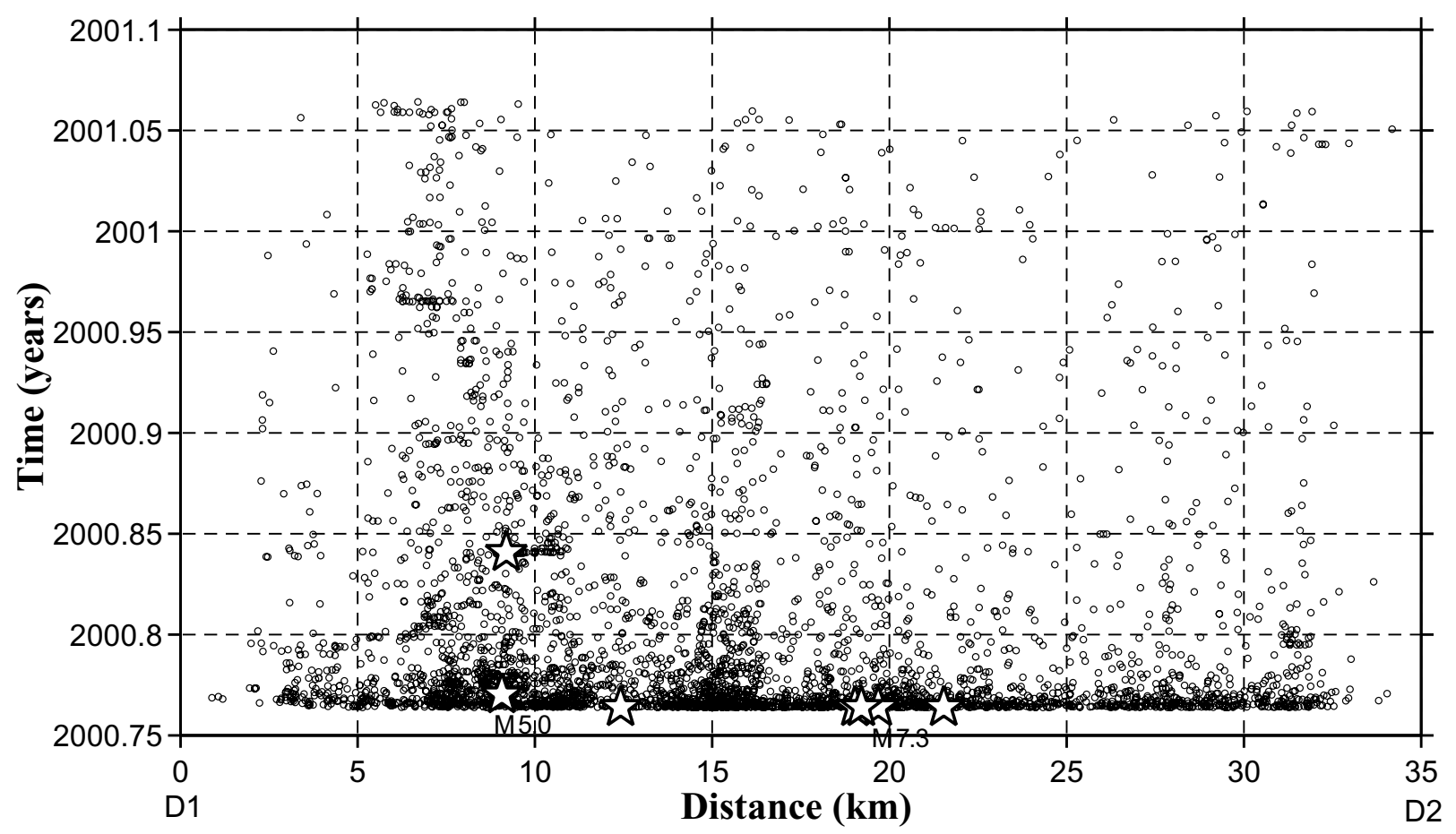

Fig. 4. Space-time diagram for the aftershocks in region A. Events are projected along the $\mathrm{D} 1\left(133^{\circ} 15.94^{\prime} \mathrm{W}, 35^{\circ} 25.93^{\prime} \mathrm{N}\right)$ to $\mathrm{D} 2\left(133^{\circ} 27.24^{\prime} \mathrm{W}\right.$, $35^{\circ} 10.41^{\prime} \mathrm{N}$ ) direction shown in Fig. $1(\mathrm{~b})$. The earthquakes with $\mathrm{M} \geq 4.5$ are marked by stars. The main shock and the largest aftershock in region $\mathrm{A}$ are indicated also by "M7.3" and "M5.0", respectively.
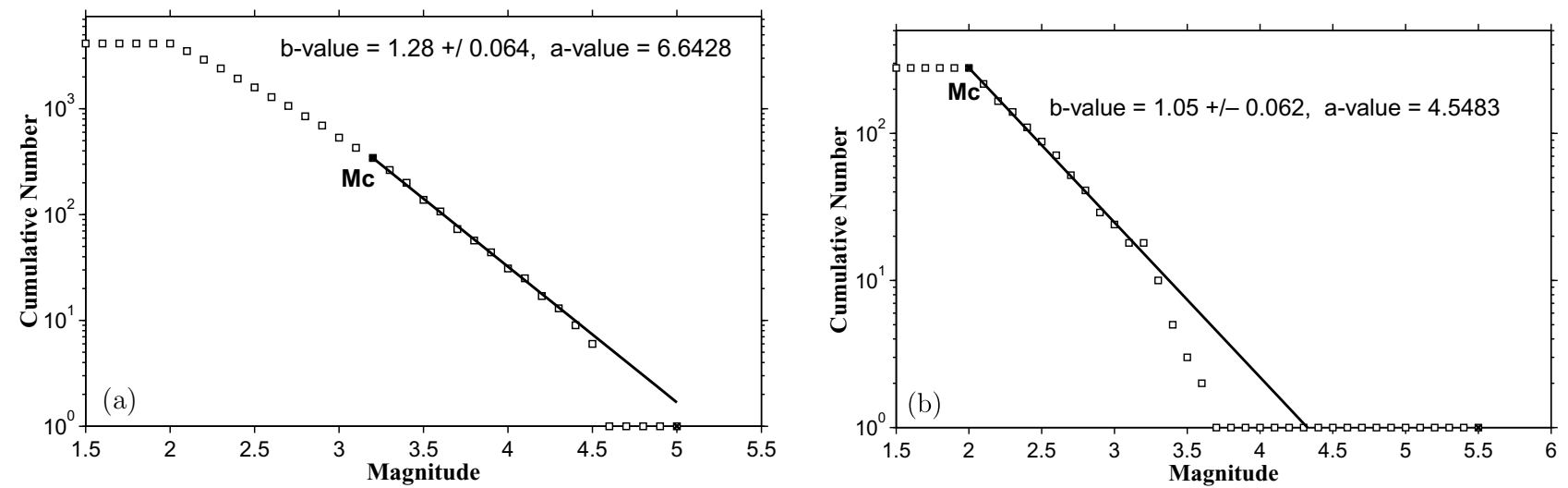

Fig. 5. Frequency-magnitude graphs for regions $\mathrm{A}$ (a) and $\mathrm{B}$ (b). The $b$-value is determined for earthquakes with $\mathrm{M} \geq \mathrm{Mc}$. Please refer to text for the method used for determining Mc. The $b$-value and its standard deviation, as well as the $a$-value in the Gutenberg-Richter relation for each region are given.

$(\mathrm{M}=5.0)$ are located NW from the main shock epicenter. Another qualitative observation can be made in Fig. 4: the earthquake frequency in the NW part (closer to D1 in Fig. 1(b)) seems to decay slower with time than in the SE region. Later (Subsection 3.2) we show that a large region in the northwest is characterized by smaller $p$-values $(\approx 0.8)$, while the region in the southwest has larger values of $p(=1-$ 1.35).

Figures 5(a) and (b) show the frequency-magnitude relation for the regions A and B, respectively. The magnitude of completeness was determined with the procedure outlined before, based on the goodness of fit of the data. We found that the completeness magnitude is 3.2 and 2.0 for the regions $\mathrm{A}$ and $\mathrm{B}$, respectively. The magnitude of completeness in region $A$ is the same as the largest value found for $\mathrm{Mc}$ as a function of time (Fig. 2). Using these magnitudes of completeness we determined the $b$-value and its standard deviation, as well as the $a$-value of the Gutenberg-Richter relation. The rather large value of $b(=1.28)$ in region $\mathrm{A}$ shows a relative abundance in small earthquakes. The $b$ value in region $B$ is close to 1.0 .

Figure 6(a) shows the occurrence rate of aftershocks in region A considering two cases: for all the events with $\mathrm{M} \geq 2.0$ and only for the events with $\mathrm{M} \geq \mathrm{Mc}(=3.2)$. $\mathrm{T}_{\text {start }}$ 

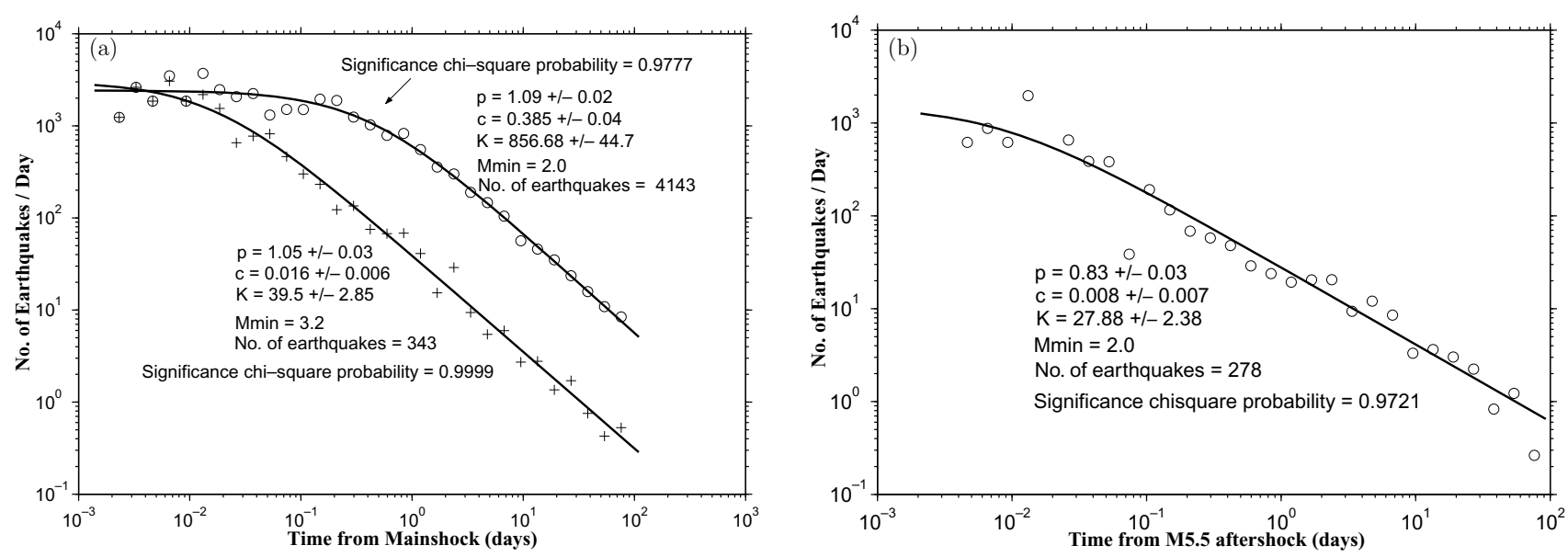

Fig. 6. (a) Occurrence rate of earthquakes versus time in region $A$ for two cases: $M \geq 2$ and $M \geq 3.2$, represented by circles and crosses, respectively. The curves represent the modified Omori formula fit to the data for the two cases. Near each distribution some relevant parameters of the fit are given: the values of $p, c$ and $K$ in the modified Omori formula, the minimum magnitude for the data, the number of earthquakes and the significance $\chi^{2}$ probability of the fit. (b) Occurrence rate of earthquakes for region B. The data are represented by circles. The same relevant parameters as for the Fig. 6(a) are given.

is assumed to be 0 in both cases. The $p, c$ and $K$ parameters were obtained using the maximum likelihood procedure and the occurrence rate was modeled by the modified Omori formula (Eq. (2)). The obtained parameters (Fig. 6(a)) have small standard deviations and the quality of fit is very good in both cases, according to the chi-square test. There is no significant difference in the $p$-value between the two cases. However, the $c$-value shows a significant difference. This result agrees well with that of Utsu et al. (1995). They pointed out that the $p$-value is independent of Mmin, but the $c$-value depends heavily on the magnitude completeness of the data. We can conclude that for the whole aftershock region the value of $p$ is close to 1 and the $c$-value is around 0.01 days. We have tested our parameters for other values of $\mathrm{M}_{\min } \geq \mathrm{Mc}$. The result does not change significantly: $p$-value remains around 1 and $c$-value is about 0.01 days or slightly less. The values obtained for Mc, $b$ and $p$ in region A are very close with those determined by the Earthquake Prediction Information Division, JMA (2001).

Figure 6(b) shows the occurrence rate of aftershocks in region $\mathrm{B}$, for events with $\mathrm{M} \geq \mathrm{Mc}(=2.0)$. The origin time corresponds to the M5.5 earthquake occurrence. The standard deviation of parameters is small and the chi-square test indicates a good fit, as shown in Fig. 6(b). The $p$-value is $0.83+/-0.03$ and $c=0.008+/-0.007$ days.

The $p$-value obtained for region $\mathrm{A}$ is close to 1 . Mogi (1962c) estimated $p$-values for 31 aftershock sequences in Japan, and discussed their geographical distribution. In general, $p$-values are high for sequences on the Japan Sea side and low on the Pacific Ocean side. Mogi (1967) thought that the aftershock activity decays faster $(p \geq 1.3)$ in regions of higher crustal temperature where the stress relaxes faster, based on the distribution of surface heat-flow values which are higher on Japan Sea side and lower on the Pacific Ocean side. The $p$-value for region A seems to be an exception, from this point of view. The $p$-value in region $\mathrm{B}$ has a relatively small value, indicating a slow decrease of earthquake rate in time. We attempt to explain this rather low value of $p$ in Subsection 3.2, when more information about the $p$-value variation in space will be available.

There are two opinions regarding the value of $c$ : one is that essentially $c$ equals to 0 , and all the reported positive $c$-values result from incompleteness in the early stage of an aftershock sequence and another is that positive $c$-values do exist. In the first case, if $c=0, n(t)$ in Eq. (2) diverges at $t=0$. According to Kagan and Knopoff (1981) this difficulty can be explained by considering that the main shock is a multiple shock composed of numerous sub-events occurring in a very short time interval. In some cases, positive $c$-values have been obtained for well-observed and selected data (see Utsu et al., 1995). Yamakawa (1968) considers that the larger $c$-value ( $c>0.01$ days) reflects more complex features of the rupture process of the main shock. In our case, the fit of the decay rate of earthquakes in region $\mathrm{A}$ indicates that $c$-value is small (about 0.01 days or less), but has probably a non-zero value. In the case of region $B$, the $c$-value is certainly very small, probably 0 or very close to 0 . Based on the results obtained for both regions $\mathrm{A}$ and $\mathrm{B}$, the $c$-value may reflect features of the rupture process, which is more complex for larger magnitude earthquakes.

So far, we presented a comparative analysis of aftershock activity in the regions A and B. The rather large area and the number of earthquakes available justifies a more in depth analysis for region A. Therefore, we have chosen two subregions A1 and A2 (Fig. 1(b)) in Region A and analyze the parameters for these two areas. The areas were chosen based on some characteristics of the seismic activity. The sub-region A1 corresponds to the areas ruptured by previous moderate events ( $\mathrm{M}$ is about 5 ) in the swarm-like activities in 1989, 1990 and 1997 (Shibutani et al., 2001). Also, this area corresponds roughly with the largest slip on the fault during the main shock (Yagi and Kikuchi, 2000; Sekiguchi and Iwata, 2000). Sub-region A2, situated in the NW portion of region $\mathrm{A}$, had not ruptured for more than 20 years before the 2000 main shock. In addition, as pointed out by Ohmi et al. (2001) and Fukuyama et al. (2001), the after- 


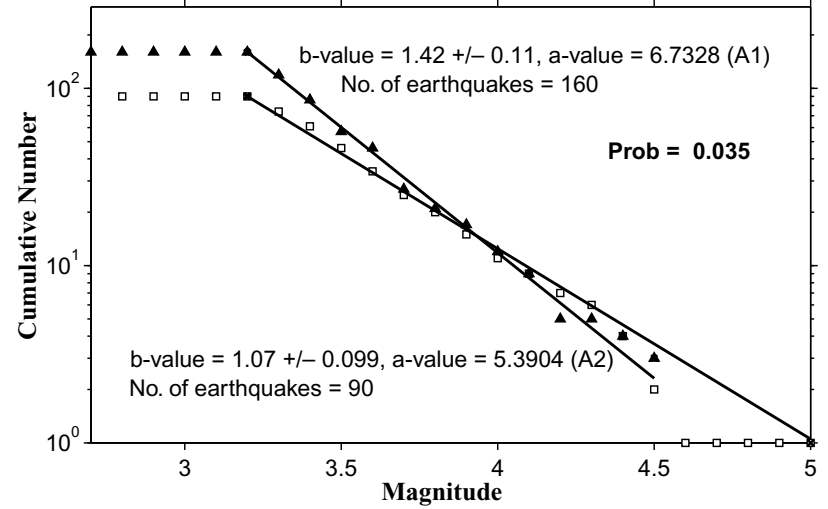

Fig. 7. Comparison between the frequency-magnitude distributions in the sub-regions, A1 and A2 in Fig. 1(b). The triangles correspond to region $\mathrm{A} 1$ and the squares to region A2. The values of $b$ and $a$ for the two sub-regions and the number of earthquakes for each sub-region are given. 'Prob' represents the probability that the two distributions come from the same population

shock activity extended towards the northwest (and also in the SE extremity) about one hour after the main shock. As shown in Fig. 1b, the seismicity in sub-region A2 is more diffuse than other parts of the aftershock area. These observations suggest that the $\mathrm{A} 1$ and $\mathrm{A} 2$ regions have different characteristics and are good candidates for comparing the aftershock activity.

Figure 7 shows the frequency-magnitude distribution for the regions $\mathrm{A} 1$ and $\mathrm{A} 2$, for events larger than or equal to 3.2 (the magnitude of completeness for region A). Region A1 is characterized by a large $b$-value of $1.42+/-0.1$, while region $\mathrm{A} 2$ has a $b$-value close to 1 . This result suggests a correlation between the rupture process and the $b$ value spatial distribution. Region A1, which experienced rupture during the recent earthquake swarms and also during the 2000 Tottori main shock is characterized by a large $b$-value, while region A2 (as well as region B studied before) has a lower value of $b$. Utsu's test confirms that the two frequency-magnitude distributions are significantly different. When checking the $p$-values for the A1 and A2 areas we could not find a significant difference between the two regions.

We tried to fit other models to the data, like the exponential model, suggested in several studies (Utsu, 1957; Mogi, 1962c; Watanabe and Kuroiso, 1970) as being more appropriate for describing the decay of aftershock activity in later periods of the sequence. For our sequence, the Omori law seems to better describe the occurrence rate decay for all the period considered. Both Utsu (1957) and Mogi (1962c) show that for about 100 days after the main shock, the decay rate is better modeled by Omori's formula for many cases. In our case the total period under consideration is about 110 days.

\subsection{Detailed spatial distribution of $b$ and $p$ values in region $\mathrm{A}$}

Figures 8(a) and (b) shows the spatial variation in $b$ and $p$ values in more detail, using the procedure described in Section 2. The grid used has a nodal separation of $0.002^{\circ}$ (about $0.2 \mathrm{~km}$ ). The closest $\mathrm{Ne}=150$ events for each node were considered and $\mathrm{Ne}_{\min }$ is taken as 100. Thus, $b$ and $p$ values for each node are computed by using between 100 and 150 earthquakes. The parameter values for nodes where the sampled earthquakes (150) are within $r=2 \mathrm{~km}$ distance from the node are represented by using a color code, on the map. Ne and $r$ can be thought as resolution parameters. An important assumption is that we chose a fixed $c=0$ days, in the modified Omori formula for the estimation of $p$-value, since $\mathrm{T}_{\text {start }}(=0.72$ days $)$ is much larger than the value of $c$ $(\approx 0.01$ days) for region $\mathrm{A}$. The magnitude of completeness was carefully checked for each node of the grid, as explained in Section 2. The magnitude of completeness varies spatially between 2.0 and 2.3, but, for most of the nodes, it has a value of 2.0. As a consequence, $b$ and $p$ values were determined using 150 events for most of the nodes. The range of values for Mc agrees with the decreased values of about 2 , observed after several hours when studying $\mathrm{Mc}$ as a function of time (Fig. 2).

The $b$ and $p$ values, obtained as described above, are shown in Figs. 8(a), and (b), respectively. The $b$-values in Fig. 8(a) vary between about 0.8 and 1.4. The goodness of fit for the frequency-magnitude distribution at each node is between $86 \%$ and $98 \%$. However, the goodness of fit is larger than or equal to $95 \%$ for most nodes. The standard deviation of $b$-values varies spatially between 0.06 and 0.13 , with most of the standard deviations less than 0.1 . The $p$ values in Fig. 8 (b) vary between 0.51 and 1.35 with standard deviations less than 0.1 .

In order to demonstrate the difference of $b$-values clearly, we have chosen two regions R1 and R2 (Fig. 8(a)), having 150 earthquakes each, and compute their frequencymagnitude distribution. The graphs, together with some relevant parameters, are presented in Fig. 9 . As can be seen, the $b$-value for region $\mathrm{R} 1$ is $0.79+/-0.06$, while $1.27+/-0.10$ for region R2. The probability that the two distributions come from the same mother population, as computed by Utsu's test, is very small $(=0.00016)$.

The differences in $p$-value for the regions R3, R4 and R5 (Fig. 8(b)) were also tested and the results are presented in Figs. 10(a) and (b). The sample in region R3 includes 633 earthquakes and its Mc equals 2.0. The $p$-value obtained for this sample is $0.86+/-0.03$. For region $\mathrm{R} 4$, we sampled 123 earthquakes, with $\mathrm{M}_{\min }=\mathrm{Mc}=2.0$, and obtained $p=1.31+/-0.07$. The graphs in Fig. 10(a) show clearly that the distributions from regions R3 and R4 are different. Also, applying the test suggested in Section 2, the difference is found significant at $95 \%$ confidence limit. In Fig. 10(b) we compare the decay of aftershock activity in the regions $\mathrm{R} 3$ and $\mathrm{R} 5$. The $p$-value for region $\mathrm{R} 5$ is $1.27+/-0.07$. Again there is a significant difference between the two distributions. Checking the decay of the occurrence rate for small samples in the NW part of the aftershock area, we found a decreased goodness of fit for the Omori formula. For this reason, we believe that some very small values of $p(0.5-0.8)$ are not determined well. Figure 10 shows that when using larger samples the $p$-value is well resolved, so there is a significant difference in $p$ values between the regions R3 and R4 and also between R3 and R5.

In the previous section we show that the area of large 

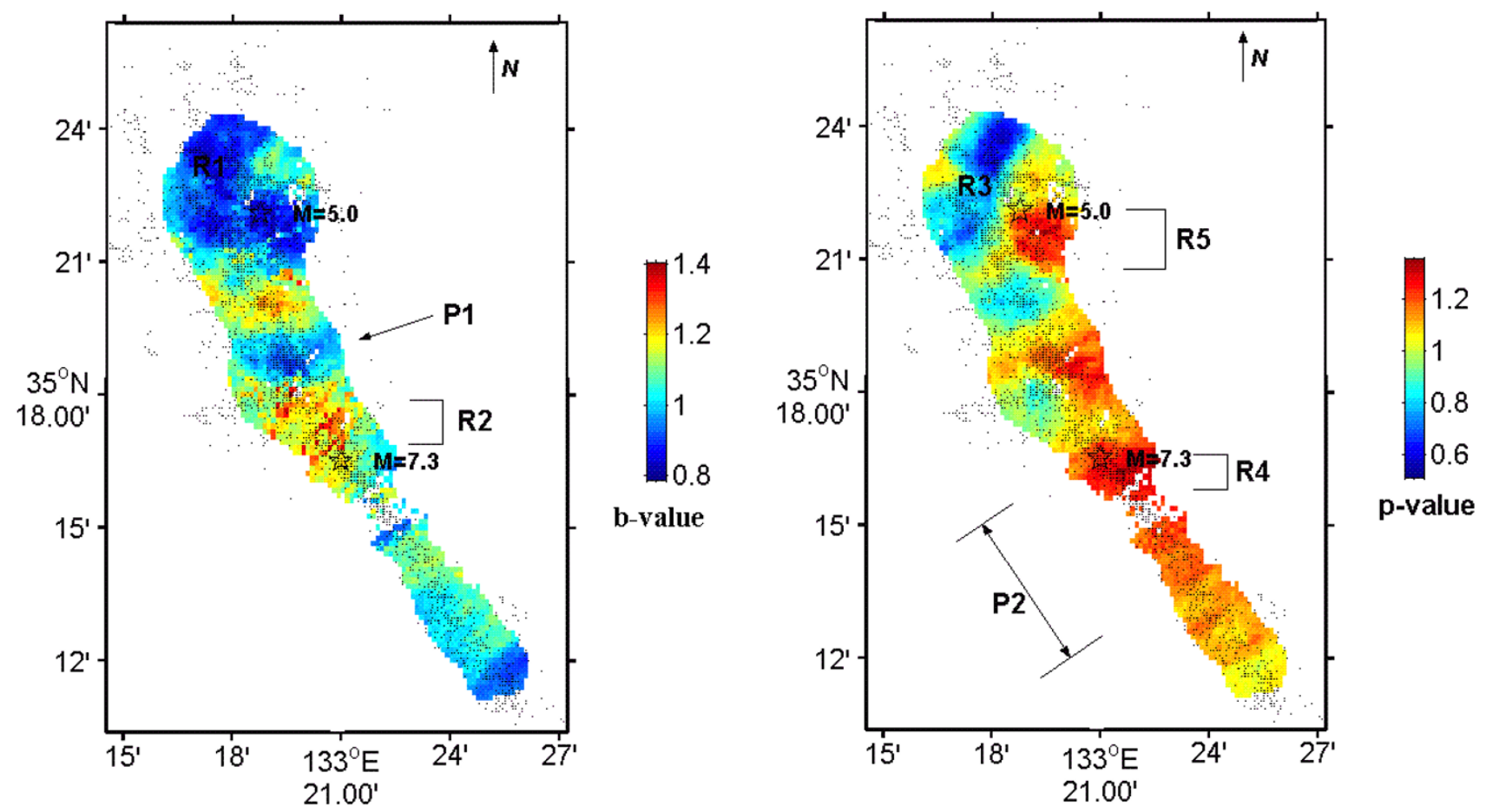

Fig. 8. (a) $b$-value map using all earthquakes with $\mathrm{M} \geq 2$ and $\mathrm{T}_{\text {start }}=0.72$ days. A $b$-value was determined by sampling the nearest 150 earthquakes for each node of a grid with nodal separation of $0.002^{\circ}$ (about $0.2 \mathrm{~km}$ ). The actual number of earthquakes used for determining the value of $b$ at each grid point is between 100 and 150, depending on the completeness of magnitude (Mc) in each node. The $b$-value was computed using the maximum likelihood method. The values of $b$ are color-coded and plotted at each node. Only the values for nodes where the sampled earthquakes are within $2 \mathrm{~km}$ distance from the node are represented. The regions R1 and R2 are used for testing the significance of the spatial variation of $b$-value (see Fig. 9 and text). P1 indicates a region with small $b$-value, as mentioned in the text. (b) A $p$-value map using the same grid and number of earthquakes in each grid-node as in the case of $b$-value map. The $p$-value was determined using a maximum likelihood method. Regions R3, R4 and R5 are used for testing the significance of the difference in $p$-value (see Fig. 10 and text). P2 indicates a region with high $p$-values.

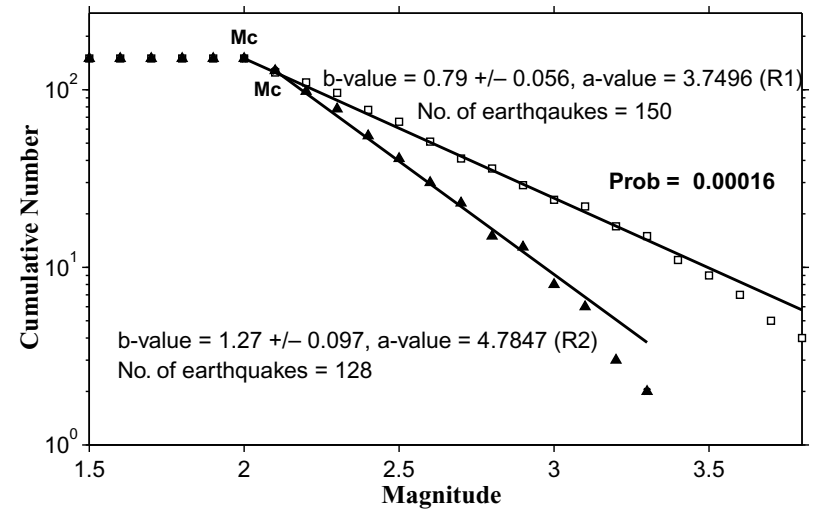

Fig. 9. Comparison between the frequency-magnitude distributions in two different regions, R1 and R2 in Fig. 8(a). The squares correspond to region $\mathrm{R} 1$ and the triangles correspond to region $\mathrm{R} 2$. The magnitude of completeness is indicated near each sample. The $b$ and $a$ values for the two samples and the number of earthquakes for each sample are given. 'Prob' represents the probability that the two distributions come from the same population.

slip during the main shock (region A1) is characterized by a large $b$-value, while the regions A2 and B have smaller values of $b$. Figure 8(a) shows $b$-values between 1.0 to 1.4 in an area extending roughly $10 \mathrm{~km}$ on both sides of the main shock, with the exception of a small patch P1 (Fig. 8(a)).
The slip distribution on the fault of the main shock for the Tottori earthquake (Yagi and Kikuchi, 2000; Sekiguchi and Iwata, 2000) shows that slip occurred approximately $10 \mathrm{~km}$ on both sides of the epicenter of the main shock, along a NW-SE oriented striking fault. Thus, the larger values of $b$ in Fig. 8(a) correlate with the region where large slip occurred during the main shock. However, largest slip occurred in the southeast region of the earthquake fault where $b$-value is not largest in value. We believe that the history of previous ruptures together with the slip during the main shock are both responsible for the distribution of $b$-value.

The patch $\mathrm{P} 1$, an "isle" of small $b$-values surrounded by larger ones, seems to correspond to a slight change in the strike of the fault of the main shock, as can be seen from the aftershock distribution. It is plausible to assume that this change may have affected the distribution of $b$ value. Probably a very detailed knowledge of the evolution of rupture process and the fault characteristics can explain such exceptions like the patch P1.

Wiemer and Katsumata (1999), by mapping the distribution of $b$-value within the M7.2 Landers, M6.7 Northridge, M6.2 Morgan Hill and M7.2 Kobe aftershock sequences, found for all four cases that the $b$-value tends to be high near the area of largest slip of the main shock. In the case of the Tottori earthquake both the history of previous ruptures and the slip during the main shock seem to be responsible for the 

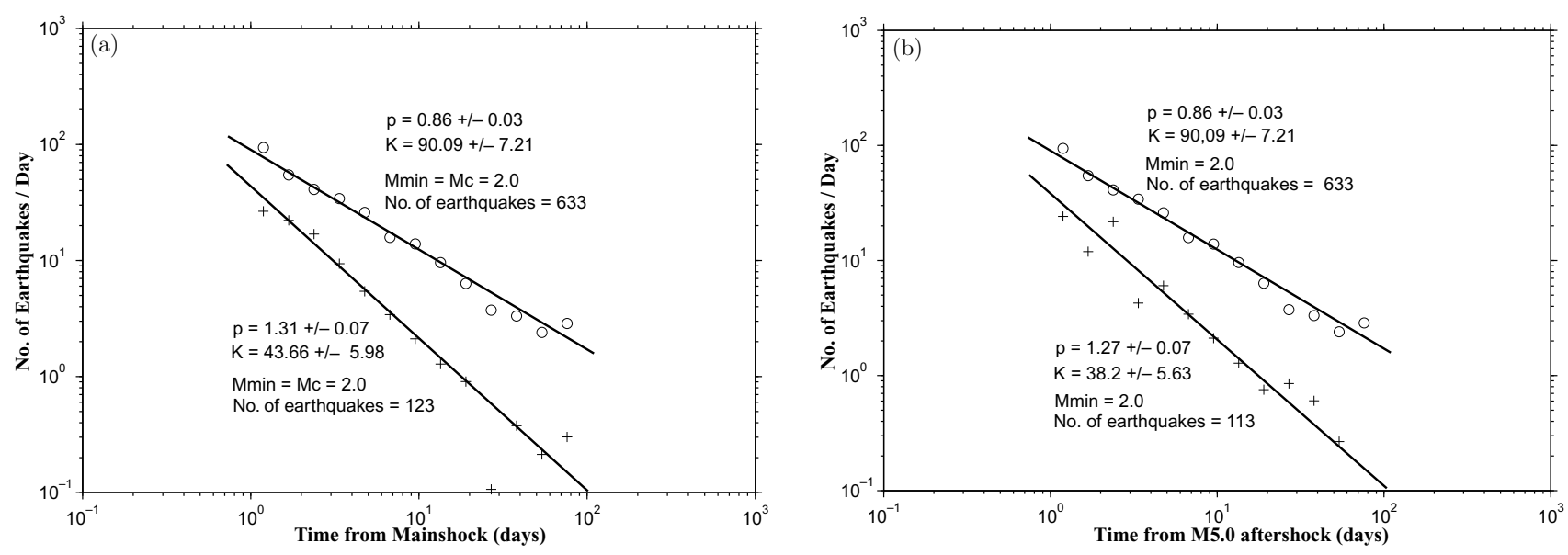

Fig. 10. Comparison between the $p$-value in the modified Omori formula for samples in the regions R3 and R4 (a) and R3 and R5 (b). The circles correspond to region R3 and the crosses to region R4 in Fig. 10(a) and R5 in Fig. 10(b). The curves represent the modified Omori formula ( $c=0$ ) fit to the data for the three cases. Near each distribution some relevant parameters of the fit are given: the values of $p$ and $K$ in the modified Omori formula, the minimum magnitude for the data and the number of earthquakes in the sample.

observed spatial patterns of $b$-value. Wiemer and Katsumata (1999), when studying the $b$-value spatial distribution for the aftershocks of Landers earthquake, found an increased $b$-value not only in the region where the slip was highest during the main shock, but also in the area that ruptured about 2 months before in the M6 Joshua Tree event, which occurred relatively close to the epicenter of Landers earthquake. Regarding the higher values of $b$ found in this study, several physical explanations are possible. One can assume that in the areas that experienced larger slip during the main shock and during previous seismic activity, the stress was reduced significantly, favoring higher $b$-values for the aftershocks. Areas with relatively low $b$-value, on the other hand, are probably regions under higher applied shear stress after the main shock. According to the Coulomb failure criterion analysis (Earthquake Prediction Information Division, JMA, 2001) both ends of the aftershock area, as well as the regions $\mathrm{B}$ and $\mathrm{C}$ have an increased stress due to the main shock. Alternatively, one can hypothesize that the areas that experienced slip are more fractured, favoring higher $b$-values (Mogi, 1962a; Main, 1996). For a more detailed discussion of various mechanisms that can affect the $b$-value (and $p$-value) spatial distribution, we refer to Wiemer and Katsumata (1999).

Mori and Abercrombie (1997) demonstrated that there is a clear depth-dependence of earthquake frequency-magnitude distributions in California, $b$-value decreasing with depth. The authors suggest that the $b$-value variation is due to decreasing heterogeneity with depth and greater stress. Shibutani et al. (2001) showed that the aftershocks of Tottori earthquake are shallower in the northwest part and also in the SE extremity of the aftershock area. If the spatial variation in $b$-value were a consequence of the depth distribution of the events, one would expect the value of $b$ to be larger in these regions, which is not the case. Therefore, the lateral variations in $b$-value are probably related to the slip distribution during the main shock and previous ruptures. However, the crustal structure may explain some details of the $b$-value spatial distribution.
There are two main regions that are characterized by higher values of $p(=1.2-1.35)$; one is the area marked as P2 in Fig. 8(b) and another one is R5 region, in the vicinity of the largest aftershock in region A (Fig. 8(b)). The former region corresponds quite clearly with the distribution of largest slip during the main shock (Yagi and Kikuchi, 2000; Sekiguchi and Iwata, 2000). Similar observations were made in the case of the Morgan Hill aftershock sequence (Wiemer and Katsumata, 1999): the volumes that experienced a high slip during the main shock have faster decay rates (i.e. larger $p$-values). They hypothesized that in some instances the frictional heating created during the main shock influences the $p$-value. Frictional heating is concentrated in high slip areas and causes a faster decay of aftershocks in these regions. This model, according to Wiemer and Katsumata (1999), is consistent with the findings of Kisslinger and Jones (1991), that describe that areas of high heat flow in the crust tend to have a higher $p$-value. In our case, the regions where the slip was large during the main shock probably also experienced such an increased frictional heating. Besides the slip during the main shock, the recent seismic activity in the region A1 may have produced also an increase in the frictional heating, which in turn favored a larger $p$-value observed in the region surrounding the main shock epicenter. However, because of the moderate size of these previous events, the associated frictional heating was probably relatively small. The relatively large value of $p$ in R5 area cannot be readily explained. The local crustal properties might be responsible for this significantly larger $p$-value. Thus, the heterogeneity of the crust may be related with the spatial variability of $p$-value (Mikumo and Miyatake, 1979; Utsu et al., 1995). It may be also possible that the occurrence of several aftershocks with $M \geq 4.3$ (the largest $M=5.0$ ) reduced the local stress, accelerating the decay of seismic activity. The large value of $p$ in $\mathrm{R} 5$ area explains why we couldn't find, in the previous section, a significant difference between the regions A1 and A2, when analyzing their $p$-values.

As we mentioned in the previous section, the $p$-value in 
the region B is significantly small. Also, as can be seen in Figs. 8(b) and 10, a large area in the NW extremity of region A is characterized by relatively small $p$-values. The activity in these regions continues with a small occurrence rate even after seven months from the main shock. We consider that this rather low decrease rate occurs in regions that did not experience significant recent ruptures.

We cannot conclude without a brief discussion on the correlation between $b$ and $p$ values. The results obtained in the previous section show that both $b$ and $p$ values are less in region $B$ than in region A. As Figs. 8(a) and 8(b) indicate, the largest $b$ and $p$ values within region $\mathrm{A}$ are found in the vicinity of the main shock, while the smallest values are found in the NW region. However, in a part of the NW region, around the large aftershock of M5.0, $p$-value is high despite of a small $b$-value. While it can be noticed that the region A1 (previously ruptured area) is generally characterized by relatively large $b$ and $p$ values, the highest values of both parameters are found in slightly different places, R2 (Fig. 8(a)) for $b$-values and R4 (Fig. 8(b)) for $p$ values, respectively. We do not have yet a clear explanation for such features, but we can speculate that they are an effect of both stress and crustal structure at a local scale (Ito and Enescu, 2001).

The significant spatial variation in both $b$ and $p$ values found in this study confirms the idea by Wiemer and Katsumata (1999) and Wiemer (2000) that the aftershock hazard after a main shock can be substantially improved if accurate information regarding the parameters spatial distribution is provided immediately following the main shock. This idea is based on the fact that the aftershock hazard depends strongly on both $b$ and $p$ values (Wiemer and Katsumata, 1999).

Finally, we would like to point out that we rigorously checked the completeness of magnitude as a function of time and space. However, we do not exclude that the Mc values are underestimated in some limited cases, especially around the epicenter of the main shock. Therefore, the values of $b$ and $p$ might be also underestimated. The correction would be an increase in these values around the main shock. However, such an increase would favor the hypothesis that large $b$ and $p$ values occur in regions that experienced larger slip during the main shock.

We believe that the spatial patterns observed for both $b$ and $p$ values are reliable and are not due to some systematic artifacts in the catalog. Our opinion is based on the stability of the results and also the fact that both JMA and Kyoto University catalogs show similar spatial distributions of the parameters.

\subsection{The seismic activity before the 2000 Western Tot- tori earthquake}

As can be seen in Fig. 1(c), the region characterized by increased seismic activity due to the occurrence of several moderate earthquakes and their aftershocks in 1989, 1990 and 1997 corresponds well with the A1 area (Fig. 1(b)). Figure 11 shows the cumulative number curve for the earthquakes in Fig. 1(c), from 1978 to the M7.3 Tottori earthquake. The threshold magnitude is 1.5 , the overall magnitude of completeness. The seismic activity is low until 1989, but starts increasing after 1989, in association with

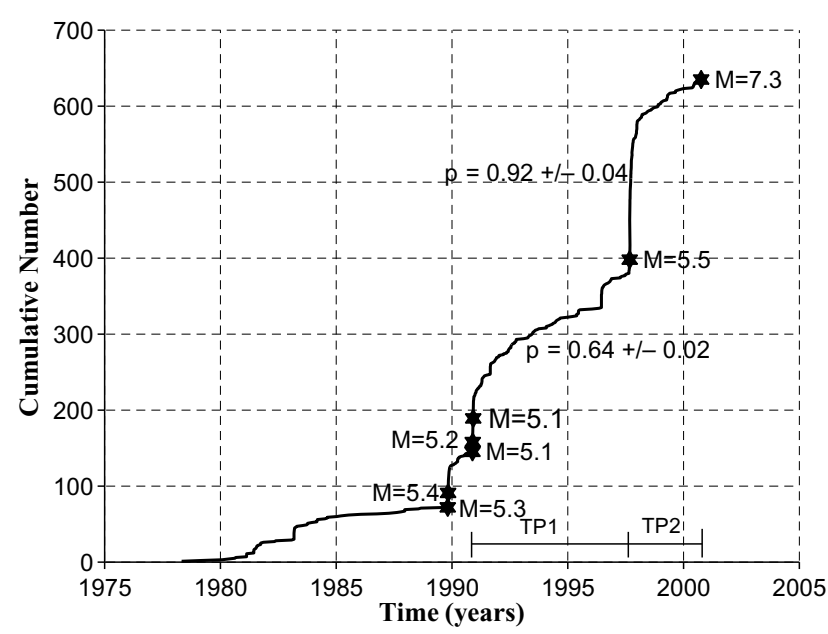

Fig. 11. Cumulative number curve of earthquakes with $\mathrm{M} \geq 1.5$ that occurred between 1978 and the 2000 Western Tottori earthquake. Stars mark the events with $\mathrm{M} \geq 5.0$. The values of $p$ given in the figure characterise the decrease rate of seismic activity in the periods TP1 and TP2, respectively, and were computed using the modified Omori formula. The magnitude of completeness for the two time periods is 1.5 and 2.5 , respectively.

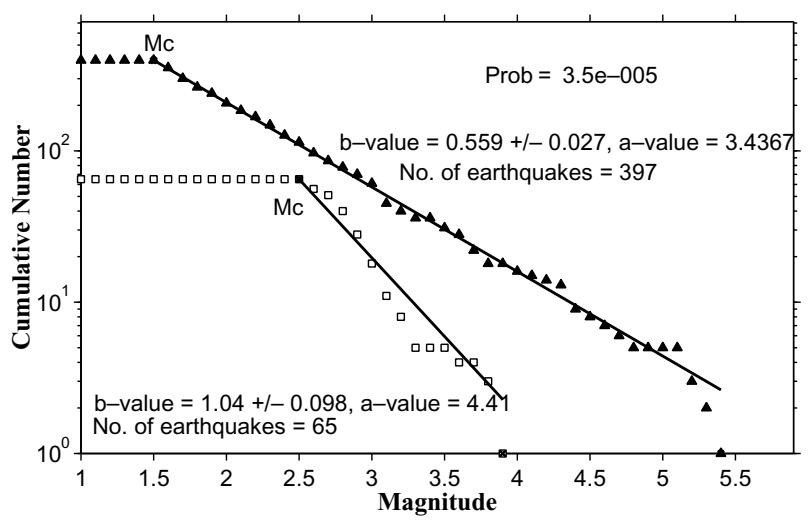

Fig. 12. Comparison of the frequency-magnitude distribution before (triangles) and after (squares) the 1997 M5.5 earthquake. Some relevant parameters for each distribution, as well as the probability that the two distributions come from the same mother population are given. "Mc" marks the magnitude of completeness (also threshold magnitude) in each case.

several moderate sized earthquakes. The aftershocks following the M5.5 earthquake in 1997 are characterized by a relatively faster decay than the aftershocks following the moderate events in 1989 and 1990. The $p$-value given after each sequence, corresponding to the time periods TP1 and TP2 (Fig. 11), supports this observation.

Figure 12 compares the frequency-magnitude distribution for the earthquakes that occurred before and after the 1997 M5.5 earthquake, taking into account the magnitude of completeness for the two periods of time. There is an increase in $b$ for the later time period and a clear difference between the two distributions. Utsu's test shows that the distributions are significantly different.

The above results suggest that the seismic activity has different characteristics before and after the M5.5 earthquake. 
The M5.5 earthquake probably ruptured the whole A1 area, while the moderate earthquakes that occurred before ruptured only parts of the same region (Shibutani et al., 2001; Matsumura, 2001). This suggests that the M5.5 event and its aftershocks "prepared" the fault for the occurrence of M7.3 Tottori earthquake. In this respect, our previous assumption (Subsections 3.1 and 3.2) that the ruptures before the Tottori earthquake have influenced the characteristics of the seismic activity, in particular the spatial distribution of $b$ and $p$ values of the aftershocks of the 2000 Tottori event, proved to be true. The results argue that, besides the rupture during the 2000 Tottori earthquake, the rupture during the 1997 M5.5 event is responsible for the large $b$ and $p$ values found in the region A1 (Subsections 3.1 and 3.2). The large incompleteness of data immediately after the Tottori earthquake does not permit an analysis of $b$-value variation in time. However, qualitatively, based on the value of $b$ before the Tottori earthquake and our work with the aftershocks of the 2000 Western Tottori earthquake, we guess that the $b$-value in region A1 continues to increase after the Tottori earthquake, reaching a stable, higher value several days after the main shock. As we have shown already, $b$ has values between 1.0 to 1.4 for aftershocks in the region A1.

\section{Conclusions}

1. The $b$-value for the main aftershock region (A), computed by using events with $\mathrm{M} \geq 3.2$ ( $\mathrm{Mc}=3.2$ ), equals to $1.28+/-0.06$. When analyzing in more detail the spatial distribution of $b$-values within region $\mathrm{A}$, the $b$-value $(1.42+/-0.11)$ is larger in an area that experienced large slip during the main shock and/or recent previous ruptures. In contrast, regions where there was no recent seismic activity and relatively small slip during the main shock have rather small values of $b$. Region $\mathrm{B}$ of triggered seismic activity is characterized by a relatively low $b$-value of $1.05+/-0.062$.

2. The modified Omori formula models well the decay of the occurrence rate in both regions A and B. Fitting the data for earthquakes with $\mathrm{M} \geq 3.2$, we obtained $p=$ $1.05+/-0.03$ and a $c$-value about 0.01 for region A. The value of $c$ is small, but seems to have a non-zero value. For region $\mathrm{B}$, the $p$-value is relatively small: $0.83+/-0.03$ and the $c$-value is probably zero or very close to zero. Within region $\mathrm{A}$ the $p$-value shows a significant spatial variability.

3 . Both $b$ and $p$ parameters show a significant change as a function of space. The $b$-value is found to vary between 0.8 and 1.4 , while $p$-value varies between 0.8 and 1.35 . These results argue that it is an oversimplification to assign one single $b$ and $p$ value to an aftershock sequence.

4. The spatial variations in the parameters $b$ and $p$ are important, and may reflect the rupture process of the main shock and/or the crustal heterogeneity in the aftershock region. Our results suggest that the rupture process of the Tottori earthquake and previous earthquake activity are the most important factors in controlling the spatial distribution of both $b$ and $p$ value. The rather large $b$-values found for areas that have ruptured recently may be explained by the relatively low stress in these regions. Areas with relatively low $b$-value, on the other hand, are probably regions under higher applied shear stress after the main shock. Alterna- tively, one can hypothesize that the areas that experienced slip are more fractured, favoring higher $b$-values. The larger $p$-values correlate with the regions that experienced large slip during main shock, while small $p$-values are found generally in regions which did not experience significant rupture. The $p$-value might be controlled by the frictional heating produced during rupture and/or the heterogeneity in the crust. Future studies are needed in order to understand better the underlying physical mechanism of the $b$ and $p$ value variation patterns.

5. The analysis of seismicity before the 2000 Western Tottori earthquake suggests that the seismic activity associated with some moderate events in 1989, 1990 and 1997 had an influence on the future seismicity in the area, in particular on the spatial distribution of $b$ and $p$ values observed for the aftershocks of Tottori earthquake. The aftershocks of the 1997 M5.5 earthquake have a larger $p$-value than previous aftershock sequences, while $b$-value has a significant increase following the M5.5 event.

6. The obtained results indicate some significant correlation between $b$-value and $p$-value, which can be summarized as follows. Both parameters are larger in region $\mathrm{A}$ than in region $B$ (conclusion nos. 1 and 2). Inside region $A$ one can find larger $b$ and $p$ values for the aftershocks of the Western Tottori earthquake around the epicenter of the main shock and smaller values for both parameters in NW of the region. As pointed out (conclusion no. 5) the $b$ and $p$ values are smaller before the 1997 event than after it. The explanation of such correlated changes relates mainly with stress changes due to experience of previous ruptures (conclusion no. 4).

Acknowledgments. The authors would like to thank Y. Ogata, S. Wiemer, J. Zhuang, T. Mizuno, T. Shibutani, K. Matsumura, H. Katao, K. Katsumata and M. Okada for helpful suggestions and comments, which improved the quality of this study. We are very grateful to J. Mori who thoroughly and critically revised the manuscript. We thank Y. Umeda and F. Takeuchi who supported and encouraged our study. We are grateful to S. Matsumura and another anonymous reviewer for very useful comments and suggestions. One of the authors (Bogdan Enescu) is grateful to the Japanese Ministry of Education for providing him a MonbuKagakusho scholarship for studying in DPRI, Kyoto University.

\section{References}

Aki, K., Maximum likelihood estimate of $b$ in the formula $\log N=a-b M$ and its confidence limits, Bull. Earthq. Res. Inst., Tokyo Univ., 43, 237239, 1965.

Earthquake Prediction Information Division, JMA, Outline of the western Tottori Prefecture earthquake in 2000, Rep. Coord. Comm. Eq. Pred., 65, 525-535, 2001 (in Japanese, with English explanation of figures).

Fukuyama, E., W. L. Ellsworth, F. Waldhauser, and A. Kubo, Very fine fault structure of the 2000 western Tottori, Japan, earthquake, Proceedings of the 2001 Japan Earth and Planetary Science Joint Meeting, Tokyo, S3$007,2001$.

Guo, Z. and Y. Ogata, Statistical relations between the parameters of aftershocks in time, space, and magnitude, J. Geophys. Res., 102(B2), $2857-$ 2873, 1997.

Gutenberg, R. and C. F. Richter, Frequency of earthquakes in California, Bull. Seismol. Soc. Am., 34, 185-188, 1944.

Ito, K. and B. Enescu, Heterogeneous structure and $b$ and $p$ values relating to the rupture of the 2000 Tottori-ken Seibu earthquake, Proceedings of the Int. Symposium on Slip and Flow processes in an below the seismogenic layer, Sendai, Japan, 78-1:78-6, 2001.

Kagan, Y. Y. and L. Knopoff, Stochastic synthesis of earthquake catalogs, J. Geophys. Res., 86, 2853-2862, 1981. 
Kisslinger, C. and L. M. Jones, Properties of aftershocks in southern California, J. Geophys. Res., 96, 11947-11958, 1991.

Main, I. G., Statistical physics, seismogenesis, and seismic hazard, Reviews of Geophysics, 34 (4), 433-462, 1996.

Matsumura, K., Seismic activity in the region around the Tottori-Shimanen border before the 2000 Tottoriken-Seibu earthquake, Special publication of DPRI, Kyoto University, dedicated to the 2000 Tottoriken-Seibu earthquake, 5-11, 2001 (in Japanese).

Mikumo, T. and T. Miyatake, Earthquake sequences on a frictional fault model with non-uniform strengths and relaxation times, Geophys. J. R. Astron. Soc., 59, 497-522, 1979.

Mogi, K., Magnitude-frequency relation for elastic shocks accompanying fractures of various materials and some related problems in earthquakes, Bull. Earthq. Res. Inst., Univ. Tokyo, 40, 831-853, 1962a.

Mogi, K., Study of the elastic shocks caused by the fracture of heterogeneous materials and its relation to earthquake phenomena, Bull. Earthq. Res. Inst., Univ. Tokyo, 40, 125-173, 1962b.

Mogi, K., On the time distribution of aftershocks accompanying the recent major earthquakes in and near Japan, Bull. Earthq. Res. Inst., Univ. Tokyo, 40, 107-124, 1962c

Mogi, K., Earthquakes and fractures, Tectonophys., 5, 35-55, 1967.

Mori, J. and R. E. Abercrombie, Depth dependence of earthquake frequency-magnitude distributions in California: Implications for rupture initiation, J. Geophys. Res., 102(B7), 15081-15090, 1997.

Ogata, Y., Estimation of the parameters in the modified Omori formula for aftershock frequencies by the maximum likelihood procedure, J. Phys. Earth, 31, 115-124, 1983.

Ohmi, S., K. Watanabe, T. Shibutani, S. Nakao, N. Hirano, A. Nakao, H. Takeuchi, F. Takeuchi, S. Miwa, A. Shito, A. Kim, and B. Enescu, The 2000 Tottori-ken Seibu earthquake - seismic activity derived from the compilation of DPRI, JMA and Hi-net data-, Bull. Disas. Prev. Res. Inst., 44, B-1, 2001.

Reasenberg, P. A., Computer programs ASPAR, GSAS and ENAS and APROB for the statistical modeling of aftershock sequences and estimation of aftershock hazard, USGS Open File Rep., 94-221, 1994.

Scholz, C. H., The frequency-magnitude relation of microfracturing in rock and its relation to earthquakes, Bull. Seismol. Soc. Am., 58, 399-415, 1968.

Sekiguchi, H. and T. Iwata, Rupture process of the 2000 Tottori-Ken Seibu earthquake using strong ground motion data, Web page: http:// sms.dpri .kyoto-u.ac.jp/iwata/ttr_e.html, 2000.

Shi, Y. and B. A. Bolt, The standard error of the magnitude-frequency $b$ value, Bull. Seismol. Soc. Am., 72(5), 1677-1687, 1982.

Shibutani, T., S. Nakao, R. Nishida, T. Fumio, K. Watanabe, and Y. Umeda, Swarm-like seismic activities in 1989, 1990 and 1997 which preceded the western Tottori earthquake (6 October 2000, $\mathrm{M}_{\mathrm{J}}=7.3$ ), Rep. Coord. Comm. Eq. Pred., 65, 576-578, 2001 (in Japanese, with English explanation of figures).

Utsu, T., Magnitude of earthquakes and occurrence of their aftershocks,
Zisin, Ser. 2, 10, 35-45, 1957 (in Japanese).

Utsu, T., A statistical study on the occurrence of aftershocks, Geophys. Mag., 30, 521-605, 1961.

Utsu, T., A method for determining the value of $b$ in formula $\log N=$ $a-b M$ showing the magnitude-frequency relation for earthquakes, Geophys. Bull. Hokkaido Univ., 13, 99-103, 1965 (in Japanese).

Utsu, T., On seismicity, in Report of the Joint Research Institute for Statistical Mathematics, Inst. for Stat. Math., Tokyo, pp. 139-157, 1992.

Utsu, T., Y. Ogata, and R. S. Matsu'ura, The centenary of the Omori formula for a decay law of aftershock activity, J. Phys. Earth, 43, 1-33, 1995.

Warren, N. W. and G. V. Latham, An experimental study of thermally induced microfracturing and its relation to volcanic seismicity, J. Geophys. Res., 75, 4455-4464, 1970.

Watanabe, H. and A. Kuroiso, Aftershocks of the earthquake of the central part of Gifu Prefecture, September 9, 1969, Bull. Earthq. Res. Inst., Univ. Tokyo, 48, 1195-1208, 1970 (in Japanese).

Wiemer, S., Introducing probabilistic aftershock hazard mapping, Geophys. Res. Lett., 27(20), 3405-3408, 2000.

Wiemer, S., A software package to analyze seismicity: ZMAP, Seis. Res. Lett., 72(2), 374-383, 2001.

Wiemer, S. and J. Benoit, Mapping the b value anomaly at $100 \mathrm{~km}$ depth in the Alaska and New Zealand subduction zones, Geophys. Res. Lett., 23, 1557-1560, 1996

Wiemer, S. and K. Katsumata, Spatial variability of seismicity parameters in aftershock zones, J. Geophys. Res., 104(B6), 13135-13151, 1999.

Wiemer, S. and M. Wyss, Mapping the frequency-magnitude distribution in asperities: An improved technique to calculate recurrence times, $J$. Geophys. Res., 102, 15115-15128, 1997.

Wiemer, S. and M. Wyss, Minimum magnitude of complete reporting in earthquake catalogs: examples from Alaska, the Western United States and Japan, Bull. Seismol. Soc. Am., 90, 859-869, 2000.

Wiemer, S. and R. F. Zuniga, ZMAP-A software package to analyze seismicity (abstract), EOS Trans. $A G U, \mathbf{7 5}(43)$, Fall Meet. Suppl., 456, 1994

Wyss, M., Towards a physical understanding of the earthquake frequency distribution, Geophys. J. R. Astron. Soc., 31, 341-359, 1973.

Wyss, M., K. Shimazaki, and S. Wiemer, Mapping active magma chambers by b values beneath the off-Ito volcano, Japan, J. Geophys. Res., 102(B9), 20413-20433, 1997.

Yagi, Y. and M. Kikuchi, Source Rupture Process of the Tottori-ken Seibu earthquake of Oct. 6, 2000, Web page: http : / / wwweic.eri . u-tokyo.ac.jp/yuji/tottori/, 2000.

Yamakawa, N., Foreshocks, aftershocks, and earthquake swarms (IV) Frequency decrease of after shocks in its initial and later stages, Pap. Met. Geophys., 19, 109-119, 1968.

B. Enescu (e-mail: benescu@rcep.dpri.kyoto-u.ac.jp) and K. Ito 